\title{
LEUCHTENDE FINSTERNIS*
}

\author{
ALFRED BÖGLI
}

Mit Farbtafel

Seit jeher übten Höhlen auf den Menschen eine Anziehungskraft aus. Die ungewohnten Formen, die gefahrd rohende Enge, das karge, ungenügende Licht machen den überblickbaren Raum zu einer seltsamen Welt, die sich unendlich weit ins Dunkel fortzusetzen scheint. Die menschliche Vorstellung ergänzt das nur Erahnte, kaum richtig Erfaßbare zu einem geheimnisvollen, bald zauberhaften, bald unheimlichen Ganzen.

Es liegt wie ein Naturgesetz im Menschen, daß er nach Erkenntnis strebt, danach, das Unbekannte zu enträtseln. Und Höhlenforschung ist ein Teil in diesem Streben. Die Rätsel einer unerforschten Höhle enthalten das Versprechen ungeahnter Wunder, den Reiz neuer Entdeckungen und die Forderung an den Einzelnen, sich voll für deren Lösung einzusetzen. Im voraus fühlt man Ehrfurcht vor den Räumen, die noch nie eines Menschen Fuß betrat, in denen noch nie die menschliche Stimme widerhallte und kein Lichtstrahl je das zeitlose Dunkel durchbrach. Daher geht von der Speläologie eine Faszination aus, die diese Wissenschaft aus dem Kreise der andern heraushebt. Sie lockt vor allem junge, begeisterungsfähige Menschen, deren Abenteuerlust das Vorrecht ihrer Jugend ist. Wenn sich das Streben nach wissenschaftlichem Erfassen und Auslegen des Geschauten hinzugesellt, dann werden die Höhlen zu einem mitreißenden Objekt exakter Forschung.

Die kleine Zahl der Wissenschafter gestattet nur einen geringen Teil der Höhlenforschungsarbeit zu bewältigen. Die Speläologie kann daher der helfenden Laien nicht entbehren, unter denen sich viele mit der Zeit eine bedeutende wissenschaftliche Ausbildung erwerben. Doch nur wenige werden so vom Feuer echten Forschens gepackt, daß sie auch dann noch ausharren, wenn die Freude am Abenteuer nicht mehr die treibende Kraft ist. Aus ihnen geht ein Stab sorgfältig arbeitender Speläologen hervor, die den Jungen Vorbild und Führer sind.

Höhlen sind überall und immer Bestandteil einer Landschaft, und die Kenntnis dieser Landschaft ist unbedingte Voraussetzung für das Verständnis der unterirdischen Hohlräume. Die Höhlenkunde ist somit 'Teil des übergeordneten Forschungsbereiches, der die gesamte Landschaft umfaßt. Doch nur in der Karstlandschaft wird die Höhle zum kennzeichnenden Merkmal, denn sie ist Ausdruck der unterirdischen Entwässerung in ihrer am weitesten entwickelten Form. Forschungsobjekt der Speläologie ist der ganze unterirdisch verkarstete Raum, ob zugänglich oder unzugänglich. Unzugängliche Bereiche werden durch die Forschung indirekt erfaßt.

Der eigentliche Wirkungsbereich ist aber der Raum über dem Karstwasser - einstige Wasserwege - die heute dauernd oder temporär zugänglich sind.

Die Speläologie ist zu einem Teil Geomorphologie, allerdings eine solche, die ihre besonderen Wege geht. In noch viel größerem $\mathrm{Maße}$ als an der Erdoberfläche müssen wir den genetischen Gehalt der Formen zu erkennen suchen. Sie sind praktisch die einzige Möglichkeit, Entstehungsvorgänge zu erfassen: denn im Zeitpunkte des Geschehens - sei es die Entwicklung aus den Primärfugen zur Höhle, seien es die Umwandlungen im phreatischen Raume, bei Hochwasser oder Einstürzen - ist die direkte Beobachtung ausgeschlossen. Deshalb ist es verständlich, daß zum Beispiel über die Kalklösung im Bergesinnern bis 1963 durchaus irrige Vorstellungen herrschten, die

* Alfred Bögli/Herbert W. Frank: Leuchtende Finsternis, Wunderwelt der Höhlen. Bildband. 160 Seiten mit to Farbtafeln, 32 einfarbigen Illustrationen, Fr. 29.- Kümmerly \& Frey, Geographischer Verlag, Bern. 
begreiflicherweise eine Unzahl ungelöster oder scheinbar unlösbarer Probleme zur Folge hatten. Erst durch die Veröffentlichung von A. Bögli über die Mischungskorrosion wurde zum Beispiel klar, daß sich die großen Höhlen nicht von außen nach innen entwickeln, sondern umgekehrt. Durch sie wurde auch die rätselhafte Kalklösung bei gänzlicher Wasserfüllung, also bei fehlender $\mathrm{CO}_{2}-Z$ ufuhr, erklärt. (Bögli : Mischungskorrosion. Ein Beitrag zum Verkarstungsproblem; «Erdkunde», Archiv für wissenschaftliche Geographie, Bd. XVIII, Lfg. 2, Bonn 1964.)

Die Höhlenkunde bedient sich vieler Wissenschaften, die für die besonderen Verhältnisse der Höhlen nicht selten Spezialgebiete entwickelt haben; Karsthydrologie, Speläobiologie und Höhlenmeteorologie sind einige Beispiele. Der Einsatz dieser Wissenszweige verrät deutlich, daß wir es in der Speläologie nicht nur mit einer analysierenden, sondern auch mit einer synthetisierenden Wissenschaft zu tun haben. Der Arbeitsbereich der Speläologie ist, auf der Grundlage der Geomorphologie, eine Synthese aller mit der Höhle verbundener Eigenschaften. Da der Synthese aber als Weg zum Erkennen und Verstehen der Einzelerscheinungen eine Analyse vorausgehen muß, so kann diese Wissenschaft der Grundlagenforschung, vor allem im Bereiche der Morphologie und der Karsthydrologie, nicht entraten. Trotzdem liegt das Endziel der Speläologie nicht darin, sondern im lückenlosen Erfassen einer Landschaft im geographischen Sinne, der Höhlenlandschaft. Infolge der Seltenheit menschlicher Eingriffe ist diese eine Naturlandschaft, was an der Gesamtkonzeption jedoch nichts ändert. Die Speläologie ist ihrem ganzen Wesen nach nicht nur ein Teil der Geomorphologie, sondern darüber hinaus eine geographische Wissenschaft.

Legende zu rechtsstehendem Farbbild:

Sinterfahnen im Medusendom des Hölloches. Sie bestehen aus reinem Kalzit und sind so dünn, $\mathrm{da} ß$ das Licht hindurchleuchtet.

\title{
DIE GEOGRAPHIE ALS KONZENTRATIONSFACH IN SCHULKOLONIEN
}

\author{
KURT BÖSIGER
}

\section{GEOGRAPHISCHE ARBEITSGEMEINSCHAFTEN AN DER MITTELSCHULE}

Noch vor 30 Jahren steckten die Schulkolonien sowohl in organisatorischer wie prinzipieller Hinsicht im Versuchsstadium (Lit. 1). Denn nur mit der Veränderung des Ortes - unter Beibehaltung der in der Schulstube üblichen Unterrichtsgestaltung sind längst nicht alle Möglichkeiten ausgeschöpft. Annaheim versuchte schon im Sommer 1935 «das Gemeinschaftsprinzip für das gesamte Leben der Kolonie einschließlich der inhaltlichen Ausprägung der Unterrichtsarbeit von Bedeutung werden zu lassen». Er wählte für seine 4. Sekundarklasse (achtes Schuljahr) die Dorfgemeinschaft von Feldis (GR), also den Alpenbauern als Nutzer und Gestalter der Naturlandschaft, als Schwerpunktthema seines Gesamtunterrichtes. Seither ist der unschätzbare Wert solcher Arbeitsgemeinschaften immer mehr erkannt worden, und die Idee der Schulkolonie hat sich in den letzten Jahren an der Oberstufe vieler Schulen mehr oder weniger durchgesetzt. Es gibt solche, welche die Schulkolonie für eine bestimmte Klassenstufe obligatorisch erklärt haben und wiederholt dieselben bewährten Lagerorte aufsuchen. An den meisten Schulen wird die Initiative den Lehrern überlassen, so auch an der 